\title{
Acquisition of Mouse Tumor Biopsies through Abdominal Laparotomy
}

\author{
Stephen A. Sastra ${ }^{1,2}$ and Kenneth P. Olive ${ }^{1,2,3}$ \\ ${ }^{1}$ Department of Medicine, Herbert Irving Comprehensive Cancer Center, Columbia University Medical Center, \\ New York, New York 10032; ${ }^{2}$ Department of Pathology, Herbert Irving Comprehensive Cancer Center, Columbia \\ University Medical Center, New York, New York 10032
}

The static analysis of tumor tissues at a single, terminal end point has been the mainstay of studies in mouse models of cancer. However, with the development of model systems that reproduce the intraand intertumoral heterogeneity of human tumors, such studies are limited by the need for large numbers of animals to overcome increased intragroup variance. The shortcomings of a single-timepoint approach to molecular analysis are especially apparent in the context of therapeutic studies, in which the dynamic response to treatment is of particular consequence. To mitigate the effects of intertumoral heterogeneity, multiple tissue samples may be harvested from the same tumor at different time points through the use of surgical biopsies. For abdominal tumors, preprocedure imaging may be used to assess the suitability of tumors for biopsy. Sterile surgical techniques are used to access the abdominal cavity, and customized instruments facilitate the immobilization and retrieval of tissue samples from hard or fibrous tumors. Thermoregulation, hemostasis, and wound closure techniques are critical to successful surgical outcomes, whereas appropriate anesthetic, analgesic, and postoperative recovery regimens are important for maintenance of animal welfare. Using a mouse model of pancreatic ductal adenocarcinoma, we present a comprehensive protocol suitable for the routine acquisition of abdominal tumor biopsies.

\section{MATERIALS}

It is essential that you consult the appropriate Material Safety Data Sheets and your institution's Environmental Health and Safety Office for proper handling of equipment and hazardous material used in this protocol.

\section{Reagents}

Absorbable gelatin compressed sponge (Gelfoam; Pfizer)

Gelfoam is a coagulant material that quickly stops bleeding and is effective for filling the space remaining after a tumor biopsy.

Buprenorphine

Buprenorphine is a controlled substance and will require appropriate federal and state licenses to purchase. In advance of the procedure, prepare a working stock of $0.03 \mathrm{mg} / \mathrm{mL}$ buprenorphine by adding a 1-mL vial of 0.3 $\mathrm{mg} / \mathrm{mL}$ buprenorphine to $9 \mathrm{~mL}$ of sterile isotonic saline.

Cleaning formula for surgical tools (detergent or Haemosol)

Disinfectant solution (e.g., chlorhexidine or an iodophor such as Povidone)

Ethanol (70\%)

\footnotetext{
${ }^{3}$ Correspondence: kenolive@ columbia.edu

(c) 2014 Cold Spring Harbor Laboratory Press

Cite this protocol as Cold Spring Harb Protoc; doi:10.1101/pdb.prot077834
} 
S.A. Sastra and K.P. Olive

Isoflurane anesthetic (see Discussion)

Isotonic saline (sterile)

Mice from GEMM of interest

This protocol uses the KrasLSL.G12D/+;p53R172H/+;PdxCre (KPC) model of pancreatic ductal adenocarcinoma as an example for demonstration purposes. Before using this protocol, it is important to become familiar with several concepts pertaining to the biopsy surgery, including anesthesia and tumor localization (see Discussion for further detail).

Ophthalmic ointment

Tissue treatment reagents, as needed (e.g., formalin, liquid nitrogen, OCT, RNAlater, etc.)

\section{Equipment}

\section{Cleaning wipes (e.g., Kimwipes)}

Cotton tip applicators (sterile)

Gauze sponges

Imaging instrumentation for preprocedure assessment (VisualSonics Vevo 2100 high-resolution ultrasound with color Doppler feature)

Medical air or oxygen supply

Microcentrifuge tubes or cryotubes for samples

Needle (27 gauge)

Surgical equipment

Anesthesia induction chamber fitted with a waste-gas scavenging system

Animal clippers (handheld) with a fine blade

Biometric system for real-time monitoring of animal vital signs (heart rate, respiration rate, core body temperature, pulse oximetry)

Biometric monitoring can include a rectal thermometer or thermocouple for core body temperature monitoring, pulse oximetry using a footpad clip sensor, and/or ECG measurement via footpad electrode connections.

Biopsy forceps

Micro biopsy forceps are a custom-made surgical instrument constructed for the purpose of firmly clamping the tumor while allowing extraction of a cylindrical core biopsy with a small diameter biopsy punch (Fig. 1). The instrument may be fabricated in a machine shop or modified from a commercially available Singley organ holding forceps (e.g., Roboz Instruments RS-5255). Two modifications are made to the Singley forceps. First, the aperture on one side of the forceps is filled in or replaced with a flat, finely serrated plate. This allows the forceps to grip the tumor firmly and provides a cutting surface for the biopsy punch to abut. Second, the aperture on the other side of the forceps is slightly enlarged to permit the passage of a 3$\mathrm{mm}$ (inner) diameter biopsy punch. The adjustment screw holds grip tension, thus allowing for greater mobility of the surgeon's off-hand.

Charcoal filters appropriate for isoflurane anesthesia waste gas scavenging

Depilatory cream (optional)

Glass plate

Isoflurane anesthesia vaporizer and manifold (e.g., VetEquip RC2 anesthesia system; see Discussion)

Micro dissecting forceps (e.g., Roboz Instruments RS-8254)

Micro dissecting retractors (e.g., Roboz Instruments RS-6510)

Micro dissecting scissors (e.g., Roboz Instruments RS-5880)

Micron tissue biopsy punch (2-mm diameter, e.g., Zivic Instruments PUN2000)

Needle holder scissors (e.g., Roboz Instruments RS-7880)

Recovery cage (heated)

Scale for weighing mice

Scalpel (No. 15 blade)

Surgical platform incorporating thermoregulated heating element

Surgical wound clip applicator (e.g., Reflex applicator and staples)

Vacuum cleaner (handheld)

Wash, autoclave, and dry heat-bead sterilize all surgical tools before use. For the surgical procedure, wear sterile surgical gloves (powder-free) and a mask, hair net, and gown. 

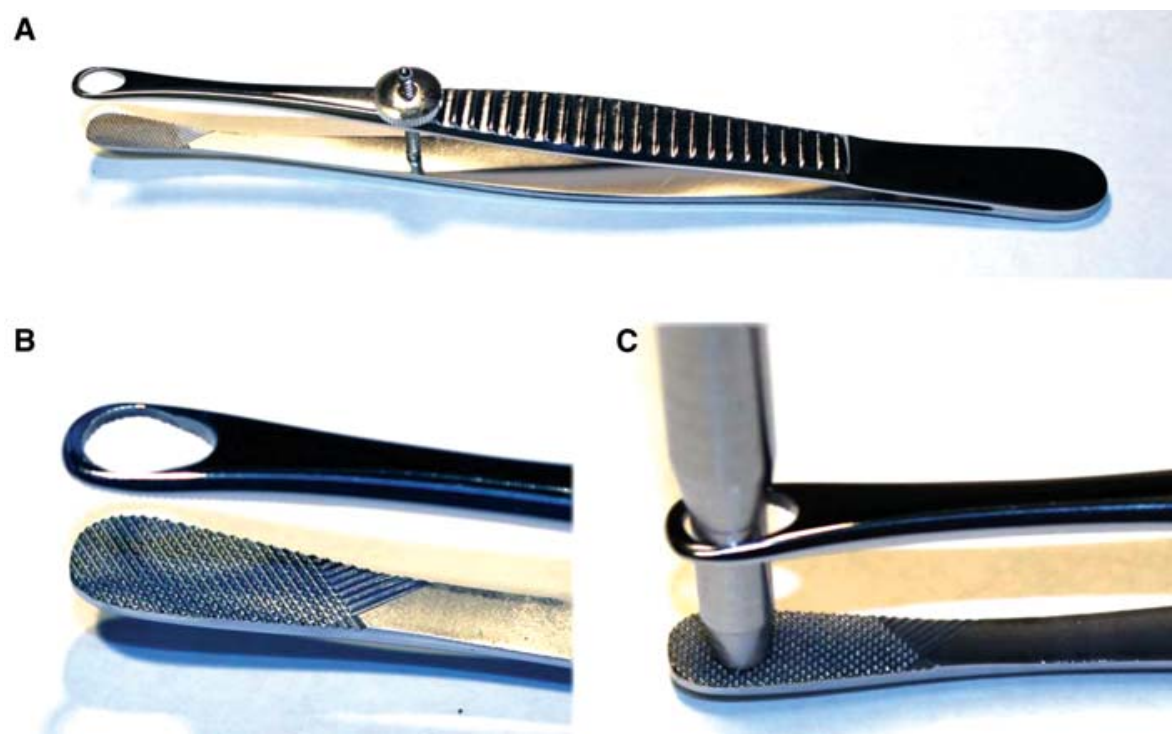

FIGURE 1. Design of biopsy forceps. (A) Biopsy forceps based on a Singley organ-holding forceps, with a filled-in aperture on one side and an enlarged aperture on the other side. $(B)$ Detail of gripping surface and aperture on biopsy forceps. (C) Demonstration of a 3-mm punch biopsy tool passing through the open aperture of biopsy forceps.

\section{Surgical suite}

The space requirements for a surgical suite are not large, but the physical layout does require careful organization. We suggest a total of five stations be set up. First, the "preprocedure station" houses mice before the start of the procedure. Second, an anesthetic "induction station" includes materials for the initial administration of anesthesia to the animal (anesthetic gas induction box or materials for injectable anesthetics). Third, the "prep station" includes materials for fur removal (clippers and handheld vacuum) and application of disinfectant surgical scrub. Fourth, the "surgical station" includes a heated platform and bright lights as well as access to an array of sterile surgical implements. Finally, the "recovery station" includes a heated cage with clean, dry bedding for postprocedure recovery.

Suture (5-0 [silk/gut]) with needle

Syringes $(1 \mathrm{~mL}$ and $5 \mathrm{~mL})$

\section{METHOD}

\section{Advance Preparation}

1. Evaluate tumor location and anatomy.

At the most basic level, this may be accomplished through manual palpation. If available, preprocedure imaging can provide valuable information on the peri-tumoral anatomy and local vascularization (Fig. 2AC). Imaging may also be used to evaluate the location of the biopsy following recovery of the animal from surgery (Fig. 2D-F). See Discussion for additional information.

2. Prepare Gelfoam coagulant plugs in advance of the surgical procedure. Perform this step in a Class II Biological Safety cabinet and wear fresh, sterile gloves. Keep sterile technique at all times.

i. Begin by cutting the Gelfoam sponge into $20-\mathrm{mm} \times 5$-mm strips.

ii. Fold each strip in half longitudinally (to 10-mm length) and gently twist between thumb and index finger to form a tight cylindrical plug (Fig. 3A).

iii. Store the plugs in individual sterile sachets. Do not autoclave the Gelfoam plugs or expose them to chemical disinfectant, as this will compromise their integrity.

3. Load a $5-\mathrm{mL}$ syringe with sterile saline for wound bathing. 

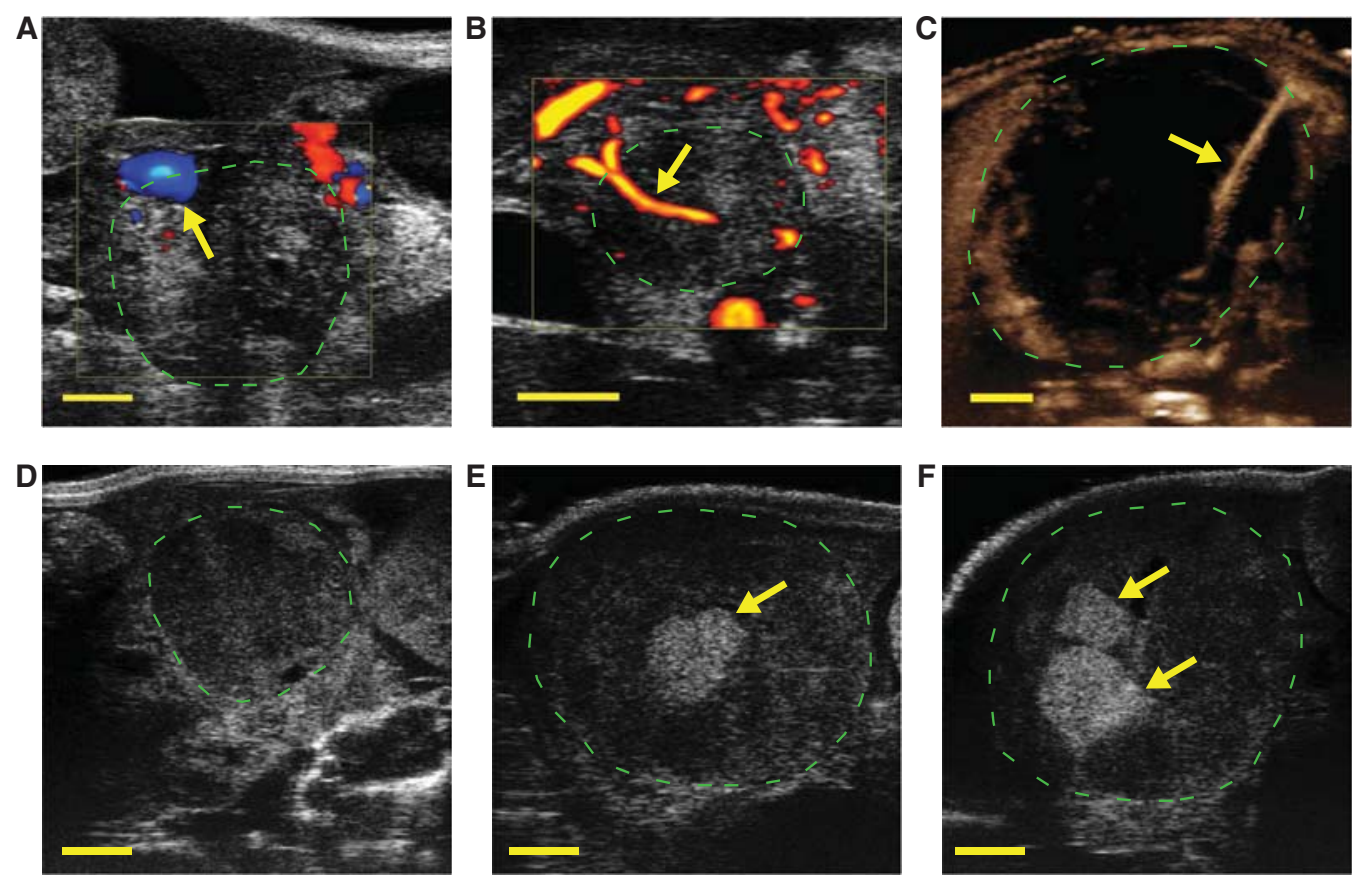

FIGURE 2. Ultrasound imaging techniques for preprocedure screening and postoperative monitoring. In each panel, a pancreatic ductal adenocarcinoma arising in a KPC mouse is imaged using a Vevo 2100 high resolution ultrasound (green hashed circle). (A) Color Doppler ultrasound imaging of a pancreatic tumor arising in a KPC mouse. Arrow indicates location of a large blood vessel on the periphery of the tumor. An approach to the tumor that avoids this vessel may make the acquisition of a biopsy feasible in this case. (B) Power Doppler ultrasound imaging identifies a moderately sized blood vessel (arrow) completely encased by the tumor, precluding retrieval of a biopsy. (C) Nonlinear contrast ultrasound is used to highlight the presence of a small blood vessel (arrow) in an otherwise poorly perfused tumor. This tumor may be operable if care is taken to avoid this vessel. $(D-F)$ A series of three B-mode highresolution ultrasound images: before surgery on Day $0(D)$, following a first biopsy surgery on Day $5(E)$, and following a second biopsy surgery on Day $11(F)$. Arrows indicate the hyperechoic (bright) signal from the Gelfoam plug at the site of each biopsy.

Animal Preparation

4. Load a 1-mL syringe (27-gauge needle) with the appropriate volume of working buprenorphine preparation.

The administration dose of buprenorphine is $0.1 \mathrm{mg} / \mathrm{kg}$ (or $120 \mu \mathrm{L}$ of the $0.03-\mathrm{mg} / \mathrm{mL}$ preparation per $25 \mathrm{~g}$ of mouse).

5. Prefill a sealed anesthesia induction chamber with $2 \%$ isoflurane in air (or oxygen).

6. Place the animal in the induction chamber until the animal shows no gross movement except for steady respiration. Confirm adequate anesthesia using the foot pad reflex test.

7. Following initial induction, reduce the isoflurane concentration to $1.5 \%$. Closely monitor the animal at all times and adjust the isoflurane concentration as needed to maintain appropriate plane of anesthesia. As the animal is moved between stations (induction, prep, or surgical), make certain to activate delivery of isoflurane to each new station and discontinue delivery of isoflurane to unused stations.

8. Move the animal to the prep station. Using fine clippers, carefully shave the fur from a large field surrounding the planned incision site (Fig. 3B). Optionally, apply depilatory cream for complete removal of fur. For a tail of pancreas tumor, clip the animal's left lateral abdominal area, from hip 
A

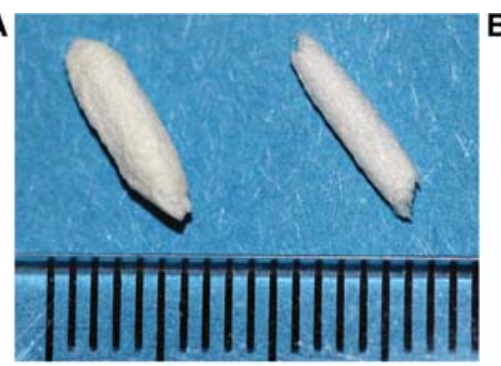

D

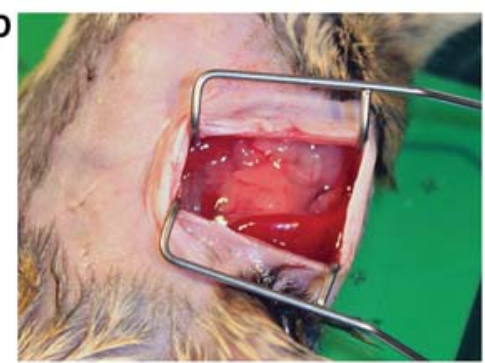

G

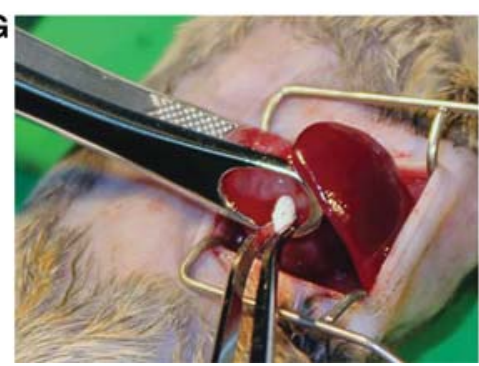

B
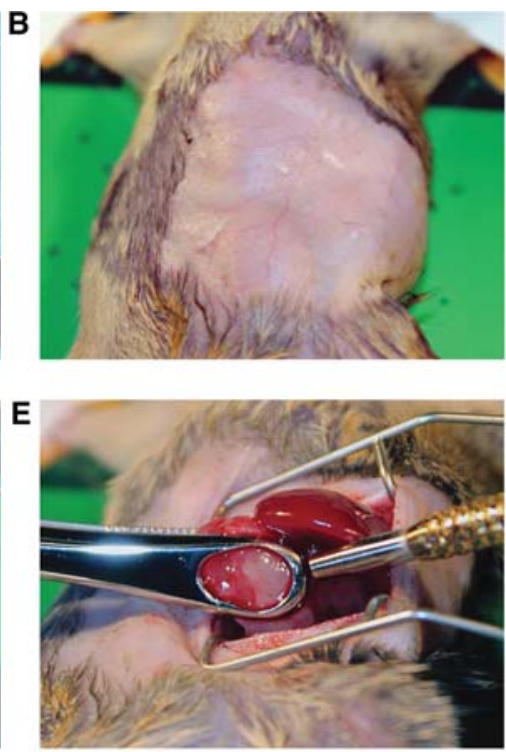

H

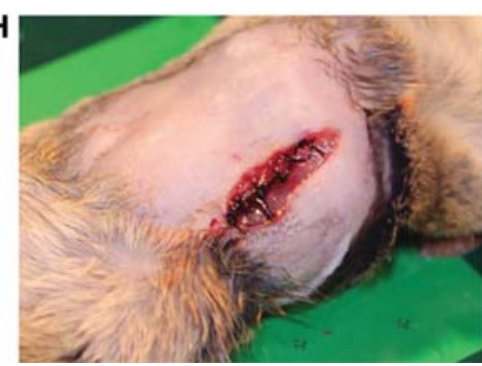

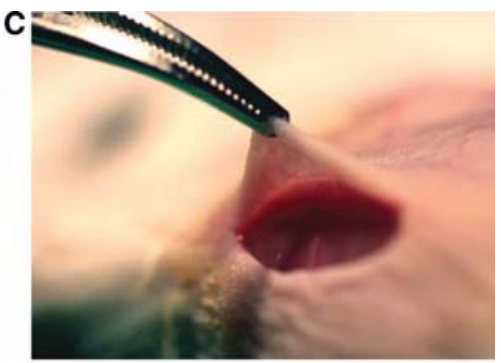
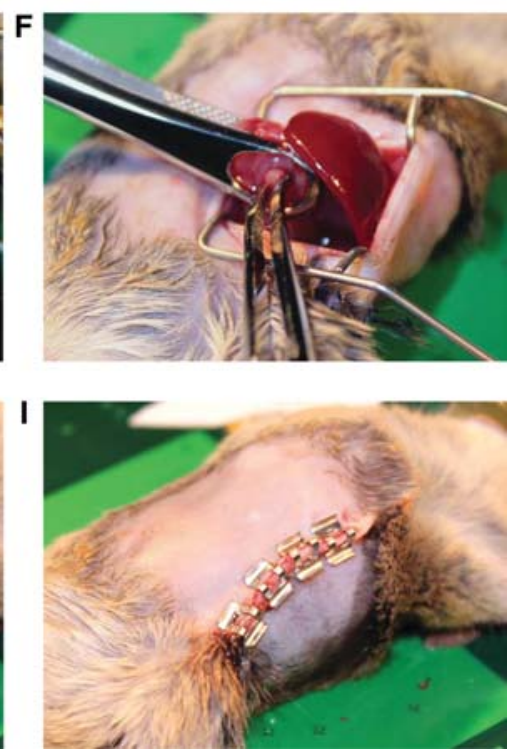

FIGURE 3. Demonstration of abdominal laparotomy procedure. (A) Rolled Gelfoam plugs are shown in millimeter scale. Plugs are used to fill the biopsy wound and encourage clotting. (B) The surgical site is prepared by removing fur and disinfection with surgical scrub. (C) A 15-mm incision is made in the skin, followed by a 12-mm incision in the abdominal wall. $(D)$ Retractors are inserted into the abdomen to create an operative field. $(E)$ The tumor is isolated and clamped in the biopsy forceps, taking care not to rupture nearby vessels. The biopsy punch tool is then passed through the tumor to the back plate of the biopsy forceps to cut a cylinder of tumor tissue. $(F)$ The biopsy core is removed with a pair of forceps. (G) A Gelfoam plug is inserted into the wound left from the biopsy. $(H)$ The abdominal wall is sutured with 5-0 silk. (I) The skin incision is repaired with surgical staples.

joint to armpit. After completion, use a handheld vacuum cleaner to collect and remove shaved fur from the Prep station, to minimize the spread of dander.

9. Apply a small amount of ophthalmic ointment to each eye to prevent dehydration.

10. Connect the biometric monitoring equipment to the animal.

11. Gently palpate the abdomen to confirm the location of the tumor and refine the location of the incision site.

12. Prepare the left flank of the animal for surgery with a scrub using disinfectant applied with a soaked gauze or cotton tip applicator, followed by a similarly applied $70 \%$ alcohol scrub. Repeat the disinfectant/alcohol scrub procedure. Always begin the scrub process at the incision line and move outward to the shaved edge. Do not go over the incision site with the same scrub.

Surgery

See Troubleshooting.

13. Administer $0.1 \mathrm{mg} / \mathrm{kg}$ of $0.03 \mathrm{mg} / \mathrm{mL}$ buprenorphine intraperitoneal (i.p.) or subcutaneous (s.c.) as a postoperative analgesic.

Preemptive administration will give the agent time to take affect before the animal's recovery. 
14. Use a scalpel or micro dissecting scissors to make a 15-mm longitudinal skin incision on the animal's left flank, centered on the latitude closest to the main tumor mass as determined by imaging and palpation (Fig. 3C).

15. Gently blunt dissect subcutaneous fascia with scissors/forceps.

16. Use a scalpel or micro dissecting scissors to make a 12-mm longitudinal peritoneal incision within the area revealed by the skin incision (Fig. 3C).

17. Use cotton tip applicators soaked in sterile saline to sponge and clean any minor wound bleeding.

18. Insert retractors into the wound and expand its edges outward to form a peritoneal opening $\sim 10$ mm wide (Fig. 3D).

19. Use micro dissecting forceps to carefully locate the tumor within the abdomen.

20. Isolate the tumor from the surrounding organs and tissues, taking care to avoid tearing or rupturing the connected tissues and vessels.

21. Expose the tumor via peritoneal opening and identify the target biopsy aperture (Fig. 3E). Take care to displace the tumor mass as little as possible during this procedure.

22. Use biopsy forceps to clamp the tumor by the axes of the target core, with the forceps aperture around one pole and the forceps plate at the opposite pole. Tighten the forceps screw to hold the tumor firmly in place (Fig. 3F).

23. Insert the biopsy punch through the forceps aperture and push through with gentle twisting until the tool passes through the tumor to the forceps plate.

24. Twist the biopsy punch against the forceps plate to cut through the distal edge of the tumor core.

25. Remove the biopsy punch and retrieve the core tissue sample (Fig. 3G). Use a razor blade or scalpel blade on a glass plate to cut the sample as required. Treat the sample as per the required collection method (e.g., formalin, liquid nitrogen, OCT, RNAlater).

26. Using micro dissecting forceps, insert a Gelfoam plug into the biopsy cavity (Fig. 3H). Hold the plug in place until the Gelfoam has expanded to fill the cavity. Add further Gelfoam plugs if necessary. Use a sterile saline-soaked cotton tip applicator to apply pressure through the aperture of the biopsy forceps to staunch bleeding.

See Troubleshooting.

27. Gently loosen the biopsy forceps screw while continuing to apply pressure with the cotton tip applicator until excessive bleeding has stopped and the tumor can be fully released.

See Troubleshooting.

28. Use sterile gauze sponges to swab the peritoneal area to remove excess blood. Bathe the area in saline. Repeat swab.

29. Release the retractors and gently replace the tumor within the abdominal space.

30. Using needle holder scissors, suture the peritoneal incision closed with 5-0 [silk]. Suture (or wound clip) the skin incision closed. Clean the wound area with sterile saline and then swab with iodine solution.

31. Remove and clean the biometric monitoring devices.

32. Remove the animal from the operating stage and lay it on its right flank on gauze in a clean, heated recovery cage without other animals.

\section{Postoperative Care}

33. Monitor the animal closely and continuously until it regains consciousness and begins walking. Monitor for signs of respiratory distress (cyanosis, irregular, or labored breathing).

34. Administer $0.5 \mathrm{~mL}$ of saline i.p. if the animal has lost significant blood during the surgery or if it shows signs of dehydration postrecovery. 
35. Following initial recovery, monitor the animal frequently over successive days.

36. Continue postoperative analgesia as advised by veterinary staff.

A typical regimen is $0.1 \mathrm{mg} / \mathrm{kg}$ of buprenorphine, administered s.c. every $12 \mathrm{~h}$ for at least $2 \mathrm{~d}$. Alternatively, $5 \mathrm{mg} / \mathrm{kg}$ of Carprofen may be administered s.c. daily.

\section{TROUBLESHOOTING}

Problem (Steps 13-32): It is difficult to maintain the animal's body temperature during surgery.

Solution: Thermoregulation is a significant risk during any rodent surgical procedure. Use of a surgical platform that incorporates a temperature-controlled heating element allows for careful control of intraoperative body temperature. Make certain that the recovery cage is also appropriately warmed.

Problem (Steps 26-27): It is difficult to maintain hemostasis during the surgical procedure.

Solution: The blood vessels surrounding a tumor are often very delicate and prone to rupture. Blood loss is the greatest risk in carrying out the surgical procedure. Care should be taken in accessing the tumor. Avoid sharp dissection of connective tissue surrounding the tumor; if access is difficult, try when possible to blunt dissection techniques. Attempt to displace the tumor from its native site as little as possible. Although Fig. 3 shows complete externalization of the tumor, it is not always necessary to do this. If it is possible to gain clear access to the tumor without externalizing, this may be preferable.

\section{DISCUSSION}

Translational cancer research is focused on the biology of tumors as they are perturbed by therapeutic agents. The dynamic response of tumors in the minutes, hours, days, and months after treatment provides important information about drug metabolism, mechanisms of action, determinants of primary drug sensitivity, and avenues of acquired drug resistance. One approach to studying these changes is to treat cohorts of animals with agents and controls in parallel and then collect tissues at a single, terminal time point. However, this single-time-point approach is predicated on the idea that the tumors arising in different animals are sufficiently similar that an effect between groups can be confidently detected with a manageable number of mice. In practice, the intertumoral heterogeneity apparent in most models is sufficiently high that only large effect sizes can be detected this way. Despite the uniformity of initiating mutations in a given cancer genetically engineered mouse model, secondary mutations will be acquired during tumor evolution that lead to at least some level of heterogeneity. Moreover, in certain models, particularly those incorporating engineered mutations that incur genomic instability (such as telomerase knockouts or point mutations in p53), inter- and intratumoral heterogeneity can be extremely high, making single-time-point approaches impractical.

One way to mitigate the effects of intertumoral heterogeneity is to retrieve tissue samples from one tumor at multiple time points. For shallow tumors such as those of the skin or mammary glands, surgical procedures to acquire tissue samples are relatively minor. Conversely, tumors arising in the thoracic cavity or in the central nervous system are extremely challenging to access in a survival setting. However, for tumors in the abdominal cavity, tumor biopsies may be efficiently acquired by laparotomy, thereby enabling a full range of paired-sample study structures. The surgical approach taken to acquire tumor biopsies from different organs will vary in relation to the local tissue anatomy, local vascular anatomy, and the physiology of the organ.

\section{Case Study: The KPC Model of Pancreatic Ductal Adenocarcinoma}

Some of the many considerations in designing a surgical approach for a specific tumor are illustrated here using the specific example of pancreatic tumors arising in the K-ras ${ }^{\text {LSL.G12D/+ }}$; p53 ${ }^{\text {LSL.R172H/+ }}$; 
Pdx1Cre ${ }^{\mathrm{tg} /+}$ (KPC) mouse model (Hingorani et al. 2005). This widely used model typically develops one or two discrete pancreatic tumors in the context of a preneoplastic pancreas. The suitability of pancreatic tumors for surgical biopsy is largely dependent on their precise location within the pancreas and their relationship to the surrounding vasculature and viscera. For example, tumors located in the head of the pancreas (HOP, on the animal's right side near the proximal duodenum) are often very difficult to biopsy because of their involvement with the duodenum, gallbladder, biliary tree, bowels, and/or retroperitoneal wall. Furthermore, these tumors are often situated near major vessels such as the aorta and vena cava and frequently invade around these structures. Apart from the challenge of isolating the tumor from these structures without tissue damage, identification of a suitable biopsy through the entire diameter of the tumor that avoids all major vessels can also be difficult. In contrast, tumors located in the tail of the pancreas (TOP, located near the spleen and left kidney) are often more amenable to surgical biopsy because this region of the pancreas is typically free of direct communication with other organs or major vessels. Although proximity to intestinal loops, the spleen, the left kidney, and the left uterine horn can complicate access, tumor isolation and core identification are significantly easier than for HOP lesions.

\section{Evaluation of Tumor Location and Vascularity}

A detailed understanding of the local tissue anatomy surrounding the site of tumor development and relevant vascular anatomy is required to plan a successful biopsy procedure. Some organs (liver, kidneys, adrenals, spleen, and ovaries) are relatively accessible, requiring only an appropriately situated peritoneal incision. However, deep organs such as the pancreas, prostate, and abdominal lymph nodes necessitate additional effort to access. Moreover, the anatomy of the tumor in relation to the remaining normal tissue of an organ also bears consideration. Tumors that extend into the lumen of an organ, such as the intestines, stomach, or bladder, are particularly challenging to access because of the need to maintain organ function and integrity. Finally, the vascular anatomy of the tumor has a critical impact on surgical outcome. Tumors often recruit new blood vessels from surrounding tissues or invade existing vessels. A poorly planned biopsy risks damage to these vessels and exsanguination.

The risk of damaging tissues and vessels can be mitigated through preprocedure imaging with a functional imaging modality that can identify vasculature. For pancreatic tumors, high-resolution ultrasound is a useful and accessible anatomical imaging technology (for a detailed protocol, see Sastra and Olive 2013). Several types of functional ultrasound can provide additional information about blood flow. Color Doppler ultrasound is sensitive to the velocity and direction of blood flow, with red color indicating movement toward the transducer and blue color indicating flow away from the transducer (Fig. 2A). Power Doppler does not indicate directionality, but is more sensitive to smaller blood vessels (Fig. 2B). In addition, contrast agents such as microbubbles (gas-filled liposomes) may be used to further highlight blood flow. Nonlinear contrast ultrasound allows the detection of contrast agents with little background signal from tissues and is the most sensitive of the three techniques for identifying small blood vessels and quantifying tissue perfusion (Fig. 2C). Together, these three technologies have proven useful for the identification of relevant vessels during preprocedure screening.

Mouse Anesthesia

We strongly encourage the use of isoflurane gas as an anesthetic agent. Isoflurane is very well tolerated by mice. As a gas, it rapidly enters the system, resulting in quick induction upon exposure and a rapid recovery upon removal. The continuous administration of a rapidly cleared agent allows for much more subtle control of plane of anesthesia compared to injected agents. The use of isoflurane anesthetic requires attention to the design of both the gas delivery and waste removal systems. Isoflurane is a liquid at room temperature that is vaporized into a mixture with air or oxygen. Ideally, the surgical suite will have three stations that require anesthesia: an induction box for initial delivery of isoflurane 
to the mice, a prep station where the mice are shaved and prepared for the procedure, and the surgical station where procedures are performed. A fourth station for postoperative recovery is also necessary, but does not require access to anesthesia. To accommodate the need for anesthesia at multiple stations, we recommend the use of a vaporizer and anesthesia system with multiple independent circuits such that gas flow can be readily manipulated to each site (e.g., the VetEquip RC2 system). At each station, passive scavenging may be achieved through the use of charcoal canisters.

The sensitivity of different mice to anesthetics will range considerably based on variables such as strain background, age, and health status; tumor-bearing mice often have increased sensitivity to isoflurane. As a general guide, initial induction should be performed with $2 \%$ isoflurane gas, followed by maintenance at $1.5 \%$. However, some animals will require as much as $2.75 \%$ isoflurane for induction or as low as $1 \%$ for maintenance. At all times, plane of anesthesia should be monitored through a combination of biophysical monitoring (pulse, heart rate, oximetry), foot pinch test, and direct observation of the animal. Veterinary staff should be consulted for appropriate training in the use of isoflurane anesthesia.

\section{Outcome}

Using the technique described above, core biopsies up to $3 \mathrm{~mm}$ in diameter may be acquired from mouse abdominal tumors, yielding up to $20 \mathrm{mg}$ of tissue (Fig. 4A). Samples may be divided longitudinally to provide half for histopathology (Fig. 4B,C) and the remainder for other molecular biology assays. The use of this biopsy surgery protocol enables the utilization of powerful paired-sample study structures that have the potential to significantly decrease the number of samples necessary to achieve appropriate statistical power. The Gelfoam plug inserted into the tumor is slowly absorbed over time. However, if it is still present at subsequent necropsy, the material may be sectioned and is easily identified histologically (Fig. 4D). Local inflammation that may be apparent immediately adjacent to the plug should be taken into consideration when acquiring necropsy tissue samples for subsequent
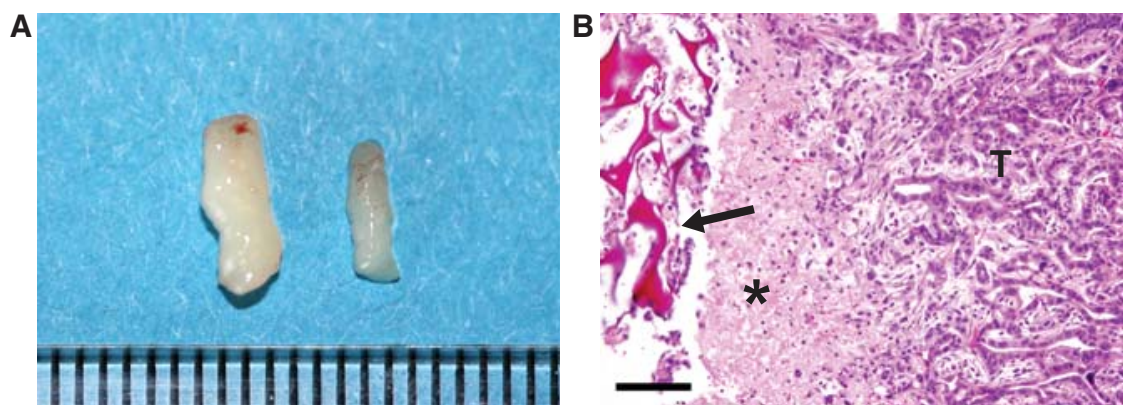

C

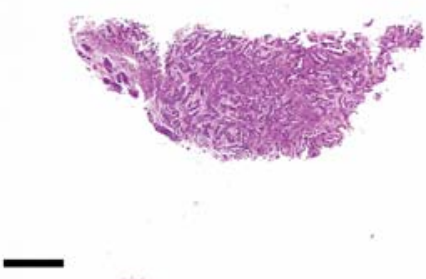

D

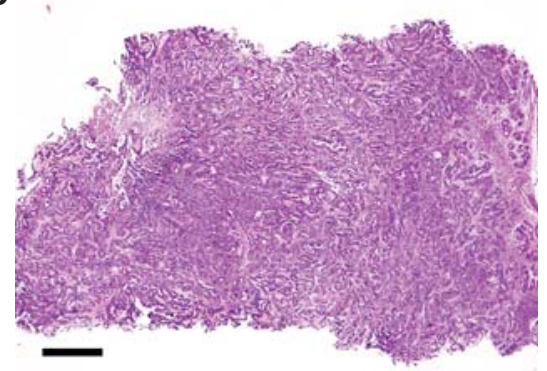

FIGURE 4. Biopsy samples may be sectioned for histology. (A) Biopsy cores of $1.5 \mathrm{~mm}$ and $3 \mathrm{~mm}$ are shown in millimeter scale. $(B)$ Hematoxylin and eosin (H\&E) stained section from a tumor that previously underwent biopsy. The Gelfoam plug is visible (arrow) as a deep red-staining, spongelike structure. Inflammatory cells (asterisk) accumulate immediately adjacent to the plug. Pancreatic ductal adenocarcinoma is apparent on the right $(T)$. (C) H\&E stained section of a 1.5-mm biopsy core. Scale bar, $500 \mu \mathrm{m}$. (D) H\&E stained section of a 3-mm biopsy core. Scale bar, $500 \mu \mathrm{m}$. 
S.A. Sastra and K.P. Olive

analyses. A vehicle-treated group should be included in all pharmacodynamic studies using this protocol to account for changes in the tumor during growth and the potential inflammatory effects of the biopsy surgery.

\section{ACKNOWLEDGMENTS}

The protocol reported here was developed with grant support from the National Institutes of Health (1R01CA157980). The authors would like to acknowledge the assistance of Carmine Palermo in the preparation of this manuscript.

\section{REFERENCES}

Hingorani SR, Wang L, Multani AS, Combs C, Deramaudt TB, Hruban RH, Rustgi AK, Chang S, Tuveson DA. 2005. Trp53R172H and KrasG12D cooperate to promote chromosomal instability and widely metastatic pancreatic ductal adenocarcinoma in mice. Cancer Cell 7: 469-483.
Sastra SA, Olive KP. 2013. Quantification of murine pancreatic tumors by high-resolution ultrasound. Methods Mol Biol 980: 249-266. 


\section{Acquisition of Mouse Tumor Biopsies through Abdominal Laparotomy}

Stephen A. Sastra and Kenneth P. Olive

Cold Spring Harb Protoc; doi: 10.1101/pdb.prot077834

\begin{tabular}{cc}
\hline $\begin{array}{r}\text { Email Alerting } \\
\text { Service }\end{array}$ & Receive free email alerts when new articles cite this article - click here. \\
\hline $\begin{array}{c}\text { Subject } \\
\text { Categories }\end{array}$ & $\begin{array}{c}\text { Browse articles on similar topics from Cold Spring Harbor Protocols. } \\
\text { Laboratory Organisms, general (923 articles) } \\
\text { Mouse (437 articles) }\end{array}$ \\
\hline
\end{tabular}

